Proc. Indian Acad. Sci. (Chem. Sci.), Vol. 92, Numbers 4 \& 5, August \& October 1983, pp. 457-462.

(C) Printed in India.

\title{
Structural evidence for the radical scavenging mechanism of some aminothiol group of radioprotectants
}

\author{
KALYANI VIJAYAN, B M VEDAVATHI and A MANI \\ Materials Science Division, National Aeronautical Laboratory, Bangalore 560017, India \\ Abstract. Aminothiols constitute an important group of radioprotectants. The structures of a \\ few well-known compounds belonging to the family of radioprotectants have been determined \\ by single crystal $\mathbf{x}$-ray diffraction methods. The sulphur and the amino nitrogen atoms are \\ separated by two tetrahedral carbon atoms in these compounds. The gauche conformation of \\ the sulphur and the nitrogen atoms and the consequent non-bonded intramolecular $S \cdots N$ \\ interaction observed in some of the crystal structures appear to favour the hypothesis that the \\ protective mechanism of these compounds is by free radical scavenging.
}

Keywords. Radioprotectants; aminothiols; crystal structure; radical scavenging; $\mathrm{S} \cdots \mathrm{N}$ interaction.

\section{Introduction}

It is well-known that on exposure to ionising radiation, biological systems incur severe damages affecting their properties and functions. Dale (1942) showed that the hazardous effects of radiation on biological systems may be reduced by administering some chemicals prior to exposure. Since then, a large number of such chemicals known as 'radioprotectants' have been identified. With the rapid advances in space technology, chemical radioprotection has gained importance and extensive research 15 being carried out to understand the mechanism of action of chemical radioprotectants Some of the well-known theories of radioprotection involve (i) the method of radical scavenging (ii) formation of mixed disulphide (iii) production of tissue hypoxia (iv) donation of hydrogen atoms (v) binding or chelating to the metal ions in the biological system and (vi) introduction of energy traps. An essential pre-requisite for understanding the mechanism of radioprotection is the study of molecular geometry of the radioprotectants. In this context, we have analysed the structures of a few radioprotectants belonging to the aminothiol family by single crystal x-ray diffraction methods. The results obtained are critically examined with reference to a particular theory of radioprotection.

\section{Aminothiol radioprotectants}

Sulphur atoms occurring in the compounds in the aminothiol family could belong to a -SH group or to a disulphide bond or could be bonded to two carbon atoms. It ${ }^{1 S}$, however, believed that the non-sulphydryl compounds are converted into the appropriate sulphydryl forms on administration and that a free sulphydryl group is essential for the radioprotective action (Shapira et al 1957; Doherty et al 1957a; Schwartz an ${ }^{d}$ Shapiro 1960; Doherty and Shapira 1963). Most aminothiol radioprotecttants contain one or more amino groups, but they could be reptaced oy guauuya gr unp 
without loss of activity. In fact, $\beta$-mercapto ethyl guanidine, a typical radioprotectant is known to be more active than $\beta$-mercapto ethylamine (Shapira et al 1957). The aminothiols have been known to possess a high degree of structural specificity with regard to their radioprotective property. In particular, the relative orientation of the amino (or guanidyl) and the thiol groups is believed to be important in their mechanism of action.

\section{X-ray results}

The bromide hydrobromide of S, 2-aminoethyl-isothiouronium (AET)(I), is an effective radioprotectant belonging to the aminothiol group. A perspective view of the AET molecule as derived from the x-ray analysis is given in figure 1 (Vijayan and Mani 1977). The terminal amino group and the sulphur atomin the AET molecule are trans about the connecting $\mathrm{C}-\mathrm{C}$ bond. This conformation eliminates the possibility of any intramolecular $\mathrm{S} \ldots \mathrm{N}$ interaction.

When administered, AET is known to undergo the chemical changes showninfigure 2

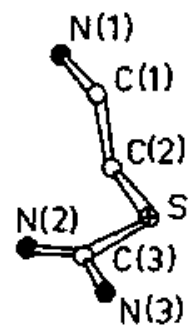

Figure 1. A perspective view of the AET molecule.

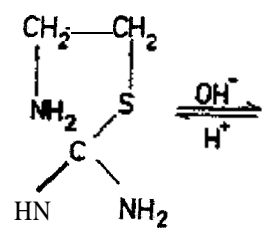

(I)<smiles>NC1(N)CCCS1</smiles>

(II)<smiles>NC(N)NCCS</smiles><smiles>[Mg]</smiles><smiles>C[14CH2]</smiles><smiles>N[C@H]1CCN2CC[C@H]12</smiles><smiles>NCCSSCCNC(N)N</smiles>

(IV)

(v)

Figure 2. Formation of 2-AT and GED from AET. 
(Khym et al 1957; Doherty et al 1957b). The two end products, viz, 2-aminothiazoline (IV) (2-AT) and bis (2-guanidinoethyl) disulphide (V) (GED), are obtained via the cyclic intermediate II, which could either open to form mercaptoethyl guanidine (III), (MEG), at $\mathrm{pH} 7$ or split offammonia to form 2-AT at $\mathrm{pH} 2 \cdot 5$. At $\mathrm{pH} 7$ and above, MEG is unstable and is readily oxidized by air to form the disulphide GED. GED, the crystal structure of which has been analysed (Vedavathi and Vijayan, to be published) is also known to protect against ionising radiation (Distefano et al 1959; Schwartz and Shapiro 1960).

A view of the GED molecule as seen along the $S-S$ bond is given in figure 3 . The two halves of the GED molecule, though chemically identical, have strikingly different structural characteristics. The sulphur and the nitrogen atoms $S(2)$ and N(4) in one half of the GED molecule are gauche, leading to an intramolecular non-bonded S . . . N distance of 3.46 A. However, in the other half of the molecule, the corresponding sulphur and nitrogen atoms $\mathrm{S}(1)$ and $\mathrm{N}(1)$ are trans about the connecting $\mathrm{C}-\mathrm{C}$ bond.

Cystamine(VI) is obtained by reduction of the well-known aminothiol radioprotect-

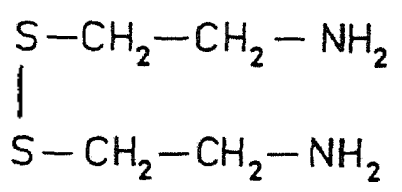

$(\mathrm{YD}$

ant cysteamine or $\beta$-mercapto ethylamine (MEA). Cystamine di $\mathrm{HCl}$, the crystal structure of which has been studied (Vedavathi and Vijayan 1979), is known to provide the same

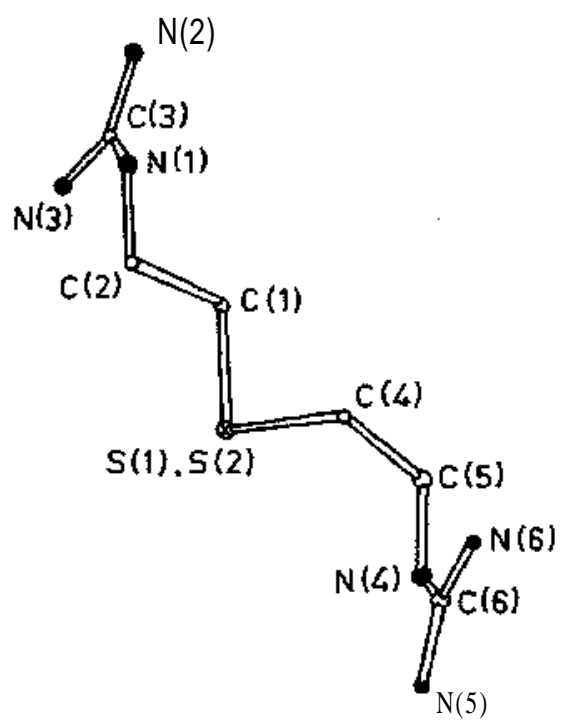

Figure 3. A view of the GED molecule as seen along the disulphide bond. 


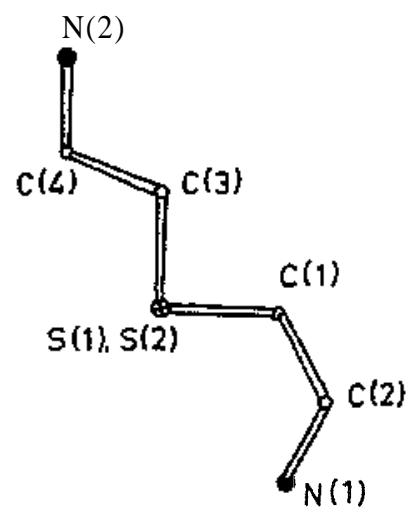

Figure 4. A view of the cystamine molecule as seen along the disulpbide bond.

order of radioprotection as cysteamine itself (Doherty et al 1957a). The cystamine molecule is essentially two ethylamine groups connected by a central disulphide bond. The only difference between the chemical structures of cystamine and GED is the replacement of ammo groups in the former by the guanidine groups in the latter. A perspective view of the cystamine molecule, as seen along the disulphide bond, is given in figure 4. As in the GED molecule, the chemically identical halves of the cystamine molecule have different structural characteristics. In one half, the sulphur and the nitrogen atoms, $\mathrm{S}(1)$ and $\mathrm{N}(1)$ are gauche leading to an intramolecular S . . N distance of 3.22 A. In the other half of the molecule, the $\mathrm{S}(2)$ and the $\mathrm{N}(2)$ atoms are trans, eliminating any possible $\mathrm{S} \ldots \mathrm{N}$ interaction.

As showninfigure 2, one of theend products obtained from AET is 2-AT.Eventhough 2-AT is known to be the toxic component, it has also been found to offer radioprotection (Distefano et al 1959; Shapira et al 1957). The crystal structure of the hydrochloride of 2-AT has also been detennined (Vedavathi and Vijayan 1981). However, the structural characteristics of 2-AT are not relevant to the present discussion.

\section{Discussion}

\subsection{S . . . N interaction in the crystal structures}

The striking, common features in the crystal structures of GED $\mathrm{Br} \bullet \mathrm{HBr}$ and cystamine di $\mathbf{H C l}$ are the gauche conformation of the sulphur and the nitrogen atoms and the consequent non-bonded S ... N interaction, observed in one half of the molecules. Similar gauche conformation and a non-bonded $\mathrm{S}$. . . N interaction have also been observed in the crystal structure of another aminothiol radioprotectant, cysteamine mono hydrochloride (MEA $\cdot \mathbf{H C l}$ ), (Jandacek and Swartz 1970). The repeated occurrence of the non-bonded $\mathrm{S}$... N interaction in the three radioprotectants strongly suggests that these features may be related to their mechanism of protection.

\subsection{Radical scavenging mechanism and $S \ldots N$ interaction}

The radical scavenging mechanism is based on the indirect action theory of radiation. It 
is well-known that radiolysis of water produces the radicals $\mathrm{H}^{\circ}, \mathrm{OH}^{\circ}$ and $\mathrm{OOH}^{\circ}$, which are free to interact with the biological system exposed to radiation and cause radiation damage. However, according to the radical scavenging theory, when a chemical radioprotectant such as an aminothiol is included in the system exposed to radiation, the radioprotectant preferentially competes for the radiation induced radicals from water, before the free radicals enter into any further action.

Doherty and Shapira (1957) have studied the radioprotective property of several aminothiols and have put forth the hypothesis that both cysteamine and cystamine are protective by virtue of their interaction with the radiation induced free radicals leading to the formation of resonance stabilised structures. According to them, the radical scavenging action of the aminothiol, say, cysteamine, (MEA), takes place as follows. The radioprotectant interacts with the free radicals $\mathrm{HOO}^{\circ}$ and $\mathrm{OH}^{\circ}$ to produce the radicals of MEA, as shown in (1).

$$
\begin{aligned}
& \mathrm{MEA}+\mathrm{HOO}^{\circ} \rightarrow \mathrm{MEA}^{\circ}+\mathrm{H}_{2} \mathrm{O}_{2}, \\
& \mathrm{MEA}+\mathrm{OH}^{\circ} \rightarrow \mathrm{MEA}^{\circ}+\mathrm{H}_{2} \mathrm{O} .
\end{aligned}
$$

The $\mathbf{M E A}^{\circ}$ radicals may enter into further interaction with the free radicals from water and regenerate into MEA itself (2).

$$
\begin{aligned}
& \mathrm{MEA}^{\circ}+\mathrm{HOO}^{\circ} \rightarrow \mathrm{MEA}+\mathrm{O}_{2}, \\
& \mathrm{MEA}^{\circ}+\mathrm{OH}^{\circ} \rightarrow \mathrm{MEA}+\mathrm{O} .
\end{aligned}
$$

The resonance structures of the radicals described in (1) are shown in figure 5.

It is obvious that in this mechanism of radical scavenging, the stability of the resonance hybrids is an important factor. This stability arises mainly from the interaction between the amino and the thiol groups, indicated by dotted lines in figure 5. The gauche conformation and the $\mathrm{S}$... N interaction observed in the crystal structures of cystamine, cysteamine and GED appear to provide evidence in support of the formation of resonance stabilised structures and thus favour the hypothesis that these compounds act by the radical scavenging mechanism.

The stability of the resonance hybrids is also controlled by the length of the carbon chain between the amino group and the sulphur atom. In cystamine, cysteamine and GED, there are only two carbon atoms between the sulphur and the nitrogen atoms thus leading to the formation of a pseudo-five-membered ring which is known to be structurally stable.

Absence of the intramolecular S . . . N interaction in the crystal structure of AET, a very effective aminothiol group of radioprotectant, is, however, consistent with the results suggesting that AET, as such, is inactive and when administered, gets converted to its active form, mercaptoethyl guanidine, MEG.

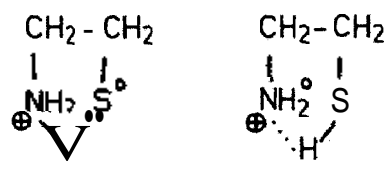

Figure 5. Resonance structures of the MEA radical. 


\section{Acknowledgement}

The authors wish to thank Dr S Ramaseshan for providing the initial impetus and encouragement.

\section{References}

Dale W M 1942 Biochem. J. 3680

Distefano V, Leary D E and Little K D 1959 J. Pharmacol. Exp. Ther. 126156

Doherty D G, Burnett W T and Shapira R 1957a Rad. Res. 713

Doherty D G and Shapira R 1963 J. Org. Chem. 281339

Doherty D G, Shapira R and Burnett W T Jr. 1957b J. Am. Chem. Soc. 795667

Jandacek R J and Swartz H M 1970 Rad. Res. 44523

Khym J X, Shapira R and Doherty D G 1957 J. Am. Chem. Soc. 795663

Schwartz E E and Shapiro B 1960 Rad. Res. 13768

Shapira R, Doherty D G and Burnett W T Jr. 1957 Rad. Res. 722

Vedavathi B M and Vijayan K 1979 Curr. Sci. 481028

Vedavathi B M and Vijayan K 1981 Acta Crystallogr. B37 475

Vedavathi B M and Vijayan K (to be published)

Vijayan K and Mani A 1977 Acta Crystallogr. B33 279 